\title{
BMJ Open Gender differences in the association of non-alcoholic fatty liver disease and metabolic syndrome with erosive oesophagitis: a cross-sectional study in a Taiwanese population
}

\author{
Wei-Chieh Hung, ${ }^{1,2}$ Jin-Shang Wu, ${ }^{2,3}$ Zih-Jie Sun, ${ }^{4,5}$ Feng-Hwa Lu, ${ }^{2,3,5}$ \\ Yi-Ching Yang, ${ }^{2,3}$ Chih-Jen Chang ${ }^{2,3}$
}

To cite: Hung W-C, Wu J-S, Sun Z-J, et al. Gender differences in the association of non-alcoholic fatty liver disease and metabolic syndrome with erosive oesophagitis: a crosssectional study in a Taiwanese population. BMJ Open 2016;6:e013106. doi:10.1136/bmjopen-2016013106

- Prepublication history for this paper is available online. To view these files please visit the journal online (http://dx.doi.org/10.1136/ bmjopen-2016-013106).

Received 20 June 2016 Revised 10 September 2016 Accepted 23 September 2016

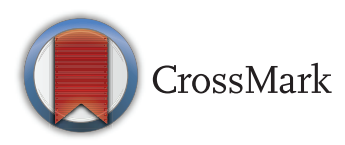

For numbered affiliations see end of article.

Correspondence to Dr Chih-Jen Chang; changcj.ncku@gmail.com

\section{ABSTRACT}

Objectives: Although metabolic syndrome correlates with erosive oesophagitis, few studies have examined the association between nonalcoholic fatty liver disease (NAFLD), associated with obesity and insulin resistance as metabolic syndrome, and erosive oesophagitis. The possible gender differences in risk factors of erosive oesophagitis should be considered. This study aimed to determine the concomitant effects of NAFLD and metabolic syndrome on erosive oesophagitis with respect to gender.

Design, setting, participants and outcome measures: This cross-sectional study, conducted between January 2000 and August 2009, included 12090 participants from the health examination center of a tertiary hospital. NAFLD was diagnosed according to ultrasonographic findings after excluding participants with excessive alcohol consumption or other liver diseases. Metabolic syndrome was determined using the revised National Cholesterol Education Program Adult Treatment Panel III criteria. Erosive oesophagitis was defined according to the Los Angeles classification by oesophagogastroduodenoscopy.

Results: On the basis of the oesophagogastroduodenoscopic findings, the prevalence of erosive oesophagitis was $20.1 \%$ $(n=1427 / 7110)$ and $9.9 \%(n=477 / 4842)$ in males and females, respectively. After adjusting for other variables, metabolic syndrome (OR 1.26; $95 \% \mathrm{Cl}$ 1.09 to 1.45 ) but not NAFLD (OR $1.14 ; 95 \% \mathrm{Cl} 0.98$ to 1.30) significantly correlated with erosive oesophagitis in males, while NAFLD (OR 1.50; 95\% $\mathrm{Cl} 1.21$ to 1.86) but not metabolic syndrome (OR 1.24; $95 \% \mathrm{Cl} 0.94$ to 1.63 ) positively correlated with erosive oesophagitis in females.

Conclusions: The detrimental effect on erosive oesophagitis is greater by metabolic syndrome than by NAFLD in males but greater by NAFLD than by metabolic syndrome in females.

\section{Strengths and limitations of this study}

- Although metabolic syndrome (MS) and nonalcoholic fatty liver disease (NAFLD) are associated with erosive oesophagitis (EO), until now there are no studies on the concomitant effects of these conditions on EO with respect to gender.

- This study reveals significant gender differences in the effects of NAFLD and MS on EO, with a greater detrimental effect on EO by MS in males and NAFLD in females.

- NAFLD positively correlates with EO in premenopausal females but not in postmenopausal females or males, suggesting that serum oestrogen levels may play an important role in EO.

- This study is cross-sectional, and thus cannot provide evidence to indicate a causal relationship between NAFLD/MS and EO with respect to gender.

\section{INTRODUCTION}

Gastro-oesophageal reflux disease involves the abnormal reflux of gastric contents into the oesophagus. Based on oesophagogastroduodenoscopic findings, the condition is classified as erosive oesophagitis (EO) or non-erosive reflux disease with or without mucosal damage. EO is a major health problem in Western countries, and epidemiological studies have demonstrated its increasing incidence. ${ }^{1}$ It affects the quality of a patient's life and increases the risk of oesophageal ulcers, peptic stricture, Barrett's oesophagus and oesophageal adenocarcinoma. ${ }^{23}$ Contributing factors to increased risk of EO include male sex, lifestyle, obesity, hiatal hernia and Helicobacter pylori negativity. $^{4-7}$ 
Metabolic syndrome (MS) is significantly associated with EO. ${ }^{6}$ The primary abnormalities of this condition are abdominal adiposity and insulin resistance. Fatty liver, presented as a macrovesicular fatty change of the liver, is also closely related to abdominal adiposity and insulin resistance. ${ }^{9}$ Park $e t a l^{10}$ examined the simultaneous effects of MS and fatty liver on EO and found that conditions are associated with EO in a Korean population. However, this earlier study did not exclude the potentially confounding effects of excessive alcohol consumption, hepatitis $\mathrm{B}$ and hepatitis C. ${ }^{10}$ Excessive alcohol consumption, which can result in EO and fatty liver, may confound the effects of fatty liver on EO. ${ }^{10} 11$ Furthermore, hepatitis B may play a role in fatty liver and $\mathrm{EO}$, while hepatitis $\mathrm{C}$ is associated with fatty liver. ${ }^{12-14}$ Non-alcoholic fatty liver disease (NAFLD) is defined as a subclass of fatty liver that is not caused by excessive alcohol consumption or other factors causing steatosis. ${ }^{15}$ Gender differences in NAFLD, MS and EO have been recently reported. ${ }^{5}{ }^{16-18}$ Sogabe et al ${ }^{16}{ }^{17}$ divided MS into visceral and subcutaneous types, with each having significantly different effects on EO; they also found that the visceral type was predominant among males, while the subcutaneous type was predominant among females. However, until now, there have been no studies regarding the concomitant effects of NAFLD and MS on EO with respect to gender. Our previous study demonstrated that NAFLD was positively correlated with EO after excluding participants with excessive alcohol drinking and hepatitis $\mathrm{B} / \mathrm{C}^{19}$ but did not further consider the effect of gender on EO and its risk factors. This study aimed to reveal any gender differences in the association of NAFLD and MS with EO.

\section{MATERIALS AND METHODS}

\section{Study subjects}

A total of 10876 male and 7341 female participants who underwent a health check-up were enrolled from the Health Examination Center of National Cheng Kung University Hospital, Taiwan between 1 January 2000 and 31 August 2009. Since this study was a secondary data analysis without any personal identification information, the requirement for informed consent was waived. We excluded participants with cancer (men/women, $n=93$ / 177), hepatitis B (men/women, $\mathrm{n}=1643 / 829)$, hepatitis C (men/women, $n=283 / 268)$, excessive alcohol drinking ( $>20 \mathrm{~g} /$ day, men/women, $\mathrm{n}=85 / 6$ ), current medications for gastrointestinal disease (men/women, $n=441 / 312$ ), current medication for arthritis (men/women, $n=67$ / 71 ), previous gastrointestinal surgery (men/women, $\mathrm{n}=7 / 5$ ) and incomplete data (men/women, $\mathrm{n}=1147$ / 831). Finally, a total of 7110 male and 4842 female participants were included for analysis.

\section{Questionnaire}

The baseline data included medical history, menopausal status (in women), medication use and lifestyle (tea drinking, smoking, alcohol consumption and habitual exercise). As previously described in detail, ${ }^{19}$ tea drinking was defined as at least once per week for at least the previous half year; smoking was defined as at least 1 pack/month for at least the previous half year; ${ }^{20}$ alcohol consumption was divided into two grades: drinker, 40 $140 \mathrm{~g} /$ week and non-drinker, $<40 \mathrm{~g}$ /week for at least the previous half year; ${ }^{4}$ and habitual exercise was defined as vigorous exercise at least 3 times/week for at least the previous half year.

\section{Anthropometric and laboratory measurements}

When standing relaxed at the end of normal expiration, waist circumference (WC) was measured midway between the lower rib border and iliac crest. ${ }^{21}$ Body mass index (BMI) was calculated as the weight $(\mathrm{kg})$ divided by the square of the height in metres $\left(\mathrm{kg} / \mathrm{m}^{2}\right)$. Obesity was defined as BMI of $\geq 27 \mathrm{~kg} / \mathrm{m}^{2}$ according to the Nutrition and Health Survey in Taiwan. ${ }^{22}$ On the basis of the WHO Asia-Pacific criteria, central obesity was defined as WC of $\geq 90 \mathrm{~cm}$ in men or $\geq 80 \mathrm{~cm}$ in women. ${ }^{23}$

A blood pressure monitor (Model 1846SX DINAMAP Monitor, Critikon, Florida, USA) was used to measure brachial systolic blood pressure and diastolic blood pressure in the supine position after at least $15 \mathrm{~min}$ of rest. According to the Seventh Report of the Joint National Committee, hypertension was defined as systolic blood pressure of $\geq 140 \mathrm{~mm} \mathrm{Hg}$ or diastolic blood pressure of $\geq 90 \mathrm{~mm} \mathrm{Hg}$ or participants who reported a history of hypertension or had undergone antihypertensive therapy. ${ }^{24}$

Blood samples were obtained from all participants in the morning following an overnight fast of at least 10 hours. The laboratory tests were conducted using a direct method with an automatic biochemical analyser (Model 7600, Hoffmann-La Roche, New Jersey, USA) and included the following parameters: fasting plasma glucose, glycosylated haemoglobin, aspartate aminotransferase, alanine aminotransferase, creatinine, total cholesterol, triglyceride and high-density lipoprotein cholesterol (HDL-C). Estimated glomerular filtration rate (eGFR) was calculated by the Modification of Diet in Renal Disease equation. ${ }^{25}$ Participants without a history of diabetes mellitus were assessed for 2 hours postload glucose level after a $75 \mathrm{~g}$ oral glucose tolerance test. Diabetes mellitus was determined on the basis of the American Diabetes Association diagnostic criteria: (1) a positive history of diabetes or current use of antidiabetic therapy, (2) fasting plasma glucose level of $\geq 126 \mathrm{mg} / \mathrm{dL} \quad(7.0 \mathrm{mmol} / \mathrm{L}), \quad$ (3) 2 hours postload glucose level of $\geq 200 \mathrm{mg} / \mathrm{dL} \quad(11.1 \mathrm{mmol} / \mathrm{L})$ and (4) glycosylated haemoglobin level of $\geq 6.5 \%{ }^{26}$ Based on the revised National Cholesterol Education Program Adult Treatment Panel III criteria for Asian populations, ${ }^{27}$ MS was diagnosed when participants presented any three of the following five risk factors: (1) central obesity; (2) systolic blood pressure of $\geq 130 \mathrm{~mm} \mathrm{Hg}$, 
diastolic pressure of $\geq 85 \mathrm{~mm} \mathrm{Hg}$, or current medication for hypertension; (3) low HDL-C as HDL-C level of $<40 \mathrm{mg} / \mathrm{dL} \quad(1.03 \mathrm{mmol} / \mathrm{L})$ for men or $<50 \mathrm{mg} / \mathrm{dL}$ ( $1.29 \mathrm{mmol} / \mathrm{L})$ for women; (4) fasting plasma glucose level of $\geq 100 \mathrm{mg} / \mathrm{dL}(5.6 \mathrm{mmol} / \mathrm{L})$ or current use of antidiabetic therapy; and (5) hypertriglyceridaemia as triglyceride level of $\geq 150 \mathrm{mg} / \mathrm{dL}(1.69 \mathrm{mmol} / \mathrm{L})$.

\section{Determination of NAFLD}

Abdominal ultrasound was performed for all participants after overnight fasting by an experienced radiologist using a $3.5 \mathrm{MHz}$ linear transducer and high-resolution ultrasonography (Xario SSA-660A; Toshiba, Nasu, Japan). Subcostal and intercostal regions were scanned and images were captured with participants in the supine position. NAFLD was diagnosed as echo findings of hepatorenal echo contrast, bright liver, deep (posterior beam) attenuation and vascular blurring. ${ }^{28}$ The degree of NAFLD was assessed by the following rule: (1) mild NAFLD: increased liver echogenicity, slightly hepatorenal contrast and relative conservation echo pattern of the walls of the portal veins; (2) moderate NAFLD: greater hepatorenal contrast, deep attenuation of echoes, loss of echoes from the walls of the portal veins and featureless appearance of the liver; and (3) severe NAFLD: obvious increased hepatorenal contrast, significantly deep attenuation of echoes and loss of most echoes from the walls of the portal veins, including the main branches. ${ }^{28}$

\section{Upper gastrointestinal endoscopy}

As previously described in detail, ${ }^{19}$ LUCERA gastroscopes (LUCERA gastroscopes; GIF-XQ260, Olympus, Tokyo, Japan) were used for oesophagogastroduodenoscopic examination, which was performed by six experienced gastroenterologists who have 15-25 years of experience to perform this examination after overnight fasting. The endoscopic classification system, as grades A, B, C and D, for EO that was proposed by the World Congress of Gastroenterology in Los Angeles was used to determine EO. ${ }^{29}$ The diagnosis of hiatal hernia was determined as diaphragmatic indentation located at $>2 \mathrm{~cm}$ distal to the Z-line (squamocolumnar junction) and the proximal margins of the gastric mucosal folds. ${ }^{30}$

\section{Statistical analyses}

The SPSS software (V.17.0; SPSS, Chicago, Illinois, USA) was used for statistical analyses. The $\chi^{2}$ and Student's t-tests were used for comparing categorical and continuous variables, where appropriate, between participants with and without EO. Multivariate logistic regression analyses were used to test whether NAFLD and MS were significant correlates of EO, and the covariates were age, hiatal hernia, eGFR, alcohol consumption, tea drinking, smoking and habitual exercise in the male group. Since no members of the female group consumed alcohol, the predictor variables were the same as those in the male group excluding alcohol consumption. Finally, we tried to examine the association between the NAFLD severity (as non, mild, moderate and severe) and the grade of EO (as A, B, and C-D). Spearman's correlation was used for univariate analysis and ordinal regression for multivariate analysis. The adjusted OR, 95\% CI and $p$ value were estimated for each variable using logistic regression analysis. A $p$ value of $<0.05$ was defined as statistically significant.

\section{RESULTS}

On the basis of oesophagogastroduodenoscopic findings, the prevalence of EO was $20.1 \% \quad(\mathrm{n}=1427 / 7110)$ and $9.9 \%(\mathrm{n}=477 / 4842)$ in males and females, respectively. The prevalences of grades A, B and C-D of EO were $16.7 \% \quad(\mathrm{n}=1187 / 7110)$ and $2.5 \% \quad(\mathrm{n}=177 / 7110)$ and $0.3 \%(\mathrm{n}=18 / 7110)$ in males, respectively. Besides, the prevalences of grades $\mathrm{A}, \mathrm{B}$ and $\mathrm{C}$ or $\mathrm{D}$ of $\mathrm{EO}$ were $8.6 \%(\mathrm{n}=414 / 4842)$ and $0.6 \%(\mathrm{n}=27 / 4842)$ and $0.02 \%$ $(\mathrm{n}=1 / 4842)$ in females, respectively. The prevalence of MS/NAFLD was $23.8 / 25.9 \% \quad(n=1694 / 7110 ; n=1839 /$ $7110)$ and $17.2 / 28.0 \%(\mathrm{n}=833 / 4842 ; \mathrm{n}=1357 / 4842)$ in males and females, respectively. In addition, the prevalence of hiatal hernia was $3.6 \%(\mathrm{n}=257 / 7110)$ and $1.5 \%$ $(\mathrm{n}=75 / 4842)$ in males and females, respectively. The prevalence of mild, moderate and severe NAFLD was $16.0 \% \quad(\mathrm{n}=1137 / 7110)$ and $9.7 \% \quad(\mathrm{n}=687 / 7110)$ and $1.8 \%(\mathrm{n}=129 / 7110)$ in males, respectively. Besides, the prevalence of mild, moderate and severe NAFLD was $17.2 \% \quad(\mathrm{n}=832 / 4842)$ and $10.7 \% \quad(\mathrm{n}=520 / 4842)$ and $0.8 \%(n=39 / 4842)$ in males, respectively. Comparison of the clinical characteristics between participants with and without $\mathrm{EO}$ is shown in table 1 . In the male group, compared with participants without EO, those with EO were older and exhibited higher levels of BMI, WC, systolic blood pressure, 2 hours postload glucose, glycosylated haemoglobin, and triglyceride and a higher prevalence of general obesity, central obesity, hypertriglyceridaemia, low HDL-C, diabetes mellitus, MS, NAFLD, hiatal hernia, tea drinkers, smokers and habitual exercisers, but a lower level of HDL-C. In the female group, participants with EO had higher BMI and WC and a higher prevalence of general obesity, central obesity, MS, NAFLD and hiatal hernia than those without EO. No participants in the female group consumed $40-140 \mathrm{~g}$ of alcohol per week.

Results of multivariate logistic regression analysis of the relationship between EO and various risk factors for male participants are shown in table 2. In model 1 , MS was significantly associated with EO (OR 1.25; 95\% CI 1.08 to 1.44) with an adjustment for age, hiatal hernia, eGFR, alcohol consumption, tea drinking, smoking and habitual exercise. Using model 2, NAFLD, substituted for MS in model 1, was not an independent risk factor for $\mathrm{EO}$ (OR 1.12; $95 \%$ CI 0.98 to 1.29). When considering MS and NAFLD simultaneously in model 3 with adjustment for the same variables in model 1 , MS was positively correlated with EO (OR $1.26 ; 95 \%$ CI 1.09 to 1.45 ) but not NAFLD 
Table 1 Comparisons of clinical variables between participants with and without EO by gender

\begin{tabular}{|c|c|c|c|c|c|c|}
\hline \multirow[b]{2}{*}{ Clinical variables } & \multicolumn{3}{|l|}{$M(n=7110)$} & \multicolumn{3}{|l|}{$F(n=4842)$} \\
\hline & $E O(n=1438)$ & non-EO $(n=5739)$ & p Value & $E O(n=484)$ & non-EO $(n=4429)$ & p Value \\
\hline Age (years) & $49.5 \pm 11.9$ & $48.4 \pm 13.0$ & 0.002 & $49.4 \pm 13.0$ & $49.0 \pm 13.1$ & 0.452 \\
\hline BMI $\left(\mathrm{kg} / \mathrm{m}^{2}\right)$ & $25.6 \pm 3.3$ & $24.9 \pm 3.2$ & $<0.001$ & $23.9 \pm 3.9$ & $23.3 \pm 3.7$ & 0.004 \\
\hline General obesity & $398(27.9)$ & 1325 (23.3) & $<0.001$ & $91(19.1)$ & $637(14.6)$ & 0.009 \\
\hline Waist circumference $(\mathrm{cm})$ & $89.5 \pm 8.7$ & $87.6 \pm 8.7$ & $<0.001$ & $78.3 \pm 9.7$ & $77.0 \pm 9.3$ & 0.005 \\
\hline Central obesity & $660(46.3)$ & $2222(39.1)$ & $<0.001$ & $182(38.2)$ & $1474(33.8)$ & 0.055 \\
\hline Systolic blood pressure $(\mathrm{mm} \mathrm{Hg})$ & $123.2 \pm 16.6$ & $122.0 \pm 15.9$ & 0.014 & $115.2 \pm 19.4$ & $113.7 \pm 17.9$ & 0.100 \\
\hline Diastolic blood pressure $(\mathrm{mm} \mathrm{Hg})$ & $73.8 \pm 10.5$ & $73.8 \pm 10.1$ & 0.858 & $66.2 \pm 10.5$ & $65.8 \pm 10.1$ & 0.347 \\
\hline Fasting plasma glucose (mmol/L) & $5.41 \pm 1.63$ & $5.36 \pm 1.49$ & 0.264 & $5.18 \pm 1.50$ & $5.16 \pm 1.30$ & 0.726 \\
\hline 2-hour postload glucose ( $\mathrm{mmol} / \mathrm{L})$ & $7.32 \pm 3.19$ & $6.96 \pm 3.11$ & $<0.001$ & $6.86 \pm 2.74$ & $6.72 \pm 2.77$ & 0.315 \\
\hline Glycated haemoglobin (\%) & $5.92 \pm 1.14$ & $5.81 \pm 1.01$ & 0.002 & $5.78 \pm 0.95$ & $5.74 \pm 0.91$ & 0.350 \\
\hline Aspartate aminotransferase (IU/L) & $27.2 \pm 17.7$ & $26.7 \pm 14.0$ & 0.232 & $23.2 \pm 10.1$ & $23.1 \pm 11.0$ & 0.895 \\
\hline Alanine aminotransferase (IU/L) & $35.8 \pm 24.4$ & $34.8 \pm 25.3$ & 0.161 & $23.6 \pm 15.4$ & $23.1 \pm 17.7$ & 0.659 \\
\hline Creatinine $(\mu \mathrm{mol} / \mathrm{L})$ & $88.8 \pm 51.6$ & $88.8 \pm 40.0$ & 1.000 & $64.5 \pm 23.0$ & $63.6 \pm 17.7$ & 0.345 \\
\hline eGFR $\left(\mathrm{mL} / \mathrm{min} / 1.73 \mathrm{~m}^{2}\right)$ & $95.9 \pm 26.1$ & $94.2 \pm 26.0$ & 0.035 & $105.5 \pm 31.1$ & $104.0 \pm 28.4$ & 0.310 \\
\hline Total cholesterol $(\mathrm{mmol} / \mathrm{L})$ & $5.15 \pm 0.95$ & $5.14 \pm 0.97$ & 0.893 & $5.13 \pm 0.99$ & $5.14 \pm 0.99$ & 0.858 \\
\hline Triglyceride (mmol/L) & $1.82 \pm 1.23$ & $1.72 \pm 1.22$ & 0.003 & $1.28 \pm 0.91$ & $1.24 \pm 0.78$ & 0.303 \\
\hline HDL-C (mmol/L) & $1.12 \pm 0.29$ & $1.16 \pm 0.30$ & $<0.001$ & $1.42 \pm 0.38$ & $1.43 \pm 0.36$ & 0.558 \\
\hline Hypertriglyceridaemia ( $\geq 1.69 \mathrm{mmol} / \mathrm{L})$ & $631(44.2)$ & $2093(36.8)$ & $<0.001$ & $97(20.3)$ & $792(18.1)$ & 0.241 \\
\hline Low HDL-C $\left(F<1.29, M<1.03 \mathrm{mmol} / \mathrm{L}^{\prime}\right)$ & 609 (42.7) & $2027(35.7)$ & $<0.001$ & $188(39.4)$ & $1648(37.8)$ & 0.497 \\
\hline Diabetes mellitus & 251 (17.6) & $838(14.7)$ & 0.008 & $69(14.5)$ & $546(12.5)$ & 0.223 \\
\hline Hypertension & $320(22.4)$ & $1241(21.8)$ & 0.632 & 95 (19.9) & $725(16.6)$ & 0.068 \\
\hline Metabolic syndrome & $421(29.5)$ & $1273(22.4)$ & $<0.001$ & $99(20.8)$ & 734 (16.8) & 0.030 \\
\hline NAFLD & $405(28.4)$ & $1434(25.2)$ & 0.015 & $173(36.3)$ & $1184(27.1)$ & $<0.001$ \\
\hline Hiatal hernia & 209 (14.6) & $48(0.8)$ & $<0.001$ & $65(13.6)$ & $10(0.2)$ & $<0.001$ \\
\hline Alcohol consumption & $11(0.8)$ & $37(0.7)$ & 0.621 & & & \\
\hline Tea drinking & 555 (38.9) & 2002 (35.2) & 0.010 & $121(25.4)$ & 1187 (27.2) & 0.394 \\
\hline Smoking & 268 (18.8) & $918(16.2)$ & 0.017 & $9(1.9)$ & $85(1.9)$ & 0.928 \\
\hline Habitual exercise & $155(10.9)$ & $504(8.9)$ & 0.020 & $26(5.5)$ & $245(5.6)$ & 0.884 \\
\hline
\end{tabular}

Data are shown as the mean \pm SD or number (\%).

BMI, body mass index; eGFR, estimated glomerular filtration rate; EO, erosive oesophagitis; F, female; HDL-C, high-density lipoprotein cholesterol; M, male; NAFLD, non-alcoholic fatty liver disease.

Table 2 Association of clinical variables with the risk of erosive oesophagitis in males*

\begin{tabular}{|c|c|c|c|c|c|c|}
\hline \multirow[b]{2}{*}{ Clinical variables } & \multicolumn{2}{|l|}{ Model 1} & \multicolumn{2}{|l|}{ Model 2} & \multicolumn{2}{|l|}{ Model 3} \\
\hline & OR (95\% Cl) & p Value & OR (95\% Cl) & p Value & OR $(95 \% \mathrm{Cl})$ & p Value \\
\hline Age (years) & 1.02 (1.01 to 1.02$)$ & $<0.001$ & 1.02 (1.01 to 1.02$)$ & $<0.001$ & 1.02 (1.01 to 1.02$)$ & $<0.001$ \\
\hline $\begin{array}{l}\text { Metabolic syndrome } \\
\text { (yes vs no) }\end{array}$ & $1.25(1.08$ to 1.44$)$ & 0.002 & & & $1.26(1.09$ to 1.45$)$ & 0.002 \\
\hline NAFLD (yes vs no) & & & 1.12 (0.98 to 1.29$)$ & 0.101 & 1.14 (0.98 to 1.30$)$ & 0.095 \\
\hline Hiatal hernia (yes vs no) & $19.88(14.42$ to 27.41$)$ & $<0.001$ & 20.03 (14.53 to 27.61$)$ & $<0.001$ & 19.75 (14.33 to 27.23$)$ & $<0.001$ \\
\hline eGFR $\left(\mathrm{mL} / \mathrm{min} / 1.73 \mathrm{~m}^{2}\right)$ & $1.01(1.00$ to 1.01$)$ & $<0.001$ & $1.01(1.01$ to 1.01$)$ & $<0.001$ & $1.01(1.00$ to 1.01$)$ & $<0.001$ \\
\hline $\begin{array}{l}\text { Alcohol consumption } \\
\text { (yes vs no) }\end{array}$ & $0.93(0.43$ to 1.98$)$ & 0.841 & $0.87(0.41$ to 1.87$)$ & 0.734 & $0.91(0.42$ to 1.94$)$ & 0.800 \\
\hline Tea drinking (yes vs no) & 1.08 (0.94 to 1.23$)$ & 0.285 & 1.07 (0.93 to 1.22$)$ & 0.344 & 1.06 (0.93 to 1.22$)$ & 0.364 \\
\hline Smoking (yes vs no) & $1.15(0.97$ to 1.35$)$ & 0.110 & $1.16(0.98$ to 1.37$)$ & 0.078 & $1.14(0.96$ to 1.35$)$ & 0.129 \\
\hline $\begin{array}{l}\text { Habitual exercise } \\
\text { (yes vs no) }\end{array}$ & 1.26 (1.03 to 1.54$)$ & 0.027 & $1.23(1.01$ to 1.51$)$ & 0.043 & 1.25 (1.02 to 1.53$)$ & 0.034 \\
\hline
\end{tabular}

\section{Adjusted OR.}

${ }^{*}$ Binary logistic regression analysis.

eGFR, estimated glomerular filtration rate; NAFLD, non-alcoholic fatty liver disease.

(OR 1.14; 95\% CI 0.98 to 1.30 ). In addition, other important factors, including age, hiatal hernia, eGFR and habitual exercise, were also demonstrated to be associated with EO.
Results of multivariate logistic regression analysis of the association between $\mathrm{EO}$ and various risk factors for female participants are shown in table 3. With adjustment for age, hiatal hernia, eGFR, tea drinking, 
Table 3 Association of clinical variables with the risk of erosive oesophagitis in females*

\begin{tabular}{|c|c|c|c|c|c|c|}
\hline \multirow[b]{2}{*}{ Variables } & \multicolumn{2}{|l|}{ Model 1} & \multicolumn{2}{|l|}{ Model 2} & \multicolumn{2}{|l|}{ Model 3} \\
\hline & OR (95\% Cl) & p Value & OR (95\% Cl) & p Value & OR (95\% Cl) & p Value \\
\hline Age (years) & $1.00(0.99$ to 1.01$)$ & 0.652 & $1.00(0.99$ to 1.01$)$ & 0.792 & $1.00(0.99$ to 1.01$)$ & 0.872 \\
\hline NAFLD (yes vs no) & 1.49 (1.21 to 1.85$)$ & $<0.001$ & & & 1.50 (1.21 to 1.86$)$ & $<0.001$ \\
\hline $\begin{array}{l}\text { Metabolic syndrome } \\
\text { (yes vs no) }\end{array}$ & & & $1.22(0.92$ to 1.61$)$ & 0.161 & $1.24(0.94$ to 1.63$)$ & 0.128 \\
\hline $\begin{array}{l}\text { Hiatal hernia } \\
\text { (yes vs no) }\end{array}$ & 65.74 (33.43 to 129.30$)$ & $<0.001$ & 67.64 (34.43 to 132.88 ) & $<0.001$ & 65.08 (33.09 to 128.00$)$ & $<0.001$ \\
\hline $\begin{array}{l}\text { eGFR }(\mathrm{mL} / \mathrm{min} / \\
\left.1.73 \mathrm{~m}^{2}\right)\end{array}$ & $1.00(1.00$ to 1.01$)$ & 0.248 & 1.00 (1.00 to 1.01$)$ & 0.645 & $1.00(1.00$ to 1.01$)$ & 0.400 \\
\hline $\begin{array}{l}\text { Tea drinking (yes vs } \\
\text { no) }\end{array}$ & $0.91(0.72$ to 1.16$)$ & 0.441 & $0.92(0.73$ to 1.17$)$ & 0.496 & $0.91(0.72$ to 1.15$)$ & 0.423 \\
\hline Smoking (yes vs no) & 1.13 (0.56 to 2.29$)$ & 0.734 & 1.15 (0.67 to 2.33$)$ & 0.697 & $1.12(0.55$ to 2.27$)$ & 0.756 \\
\hline $\begin{array}{l}\text { Habitual exercise } \\
\text { (yes vs no) }\end{array}$ & $0.93(0.59$ to 1.46$)$ & 0.746 & 0.96 (0.61 to 1.51$)$ & 0.864 & 0.93 (0.59 to 1.46$)$ & 0.761 \\
\hline
\end{tabular}

smoking and habitual exercise, NAFLD was positively related to $\mathrm{EO}$ in model 1 (OR $1.49 ; 95 \%$ CI 1.21 to 1.85). Using model 2, MS did not correlate with EO (OR 1.22; 95\% CI 0.92 to 1.61). When considering MS and NAFLD simultaneously in model 3, NAFLD (OR $1.50 ; 95 \%$ CI 1.21 to 1.86 ) but not MS (OR 1.24; $95 \%$ CI 0.94 to 1.63 ) was still associated with an increased risk of $\mathrm{EO}$ in the female group. Hiatal hernia was an additional important risk factor for EO. We divided females into premenopausal $(\mathrm{n}=2472,51.1 \%)$ and postmenopausal $(n=2370,48.9 \%)$ groups. In premenopausal females, NAFLD (OR 1.93; 95\% CI 1.43 to 2.59 ) but not MS (OR 1.20; 95\% CI 0.69 to 2.08 ) positively correlated with EO. In contrast, neither NAFLD (OR 1.15; 95\% CI 0.84 to 1.57 ) nor MS (OR $1.25 ; 95 \%$ CI 0.91 to 1.73 ) was an independently associated factor for EO in postmenopausal females (data not shown in the table).

Finally, we examined the association between NAFLD severity and EO severity in either gender. In univariate analysis, there was a slight positive correlation between the severity of NAFLD and the grade of EO (males: Spearman's correlation coefficient $=0.028, \quad \mathrm{p}=0.020$; females: Spearman's correlation coefficient $=0.038$, $\mathrm{p}=0.008$ ). In multivariate analysis, based on ordinal regression, MS was positively associated with the grade of EO $(\mathrm{OR}=1.201,95 \%$ CI 1.039 to $1.387, \mathrm{p}=0.013)$, but the severity of NAFLD was not. In females, although mild NAFLD was related to the grade of $\mathrm{EO}(\mathrm{OR}=1.385,95 \%$ CI 1.0690 to $1.793, \mathrm{p}=0.013)$, MS, moderate and severe NAFLD were not (data not shown).

\section{DISCUSSION}

Our results indicate significant gender differences in the effects of NAFLD and MS on EO. Subgroup analysis with respect to gender demonstrated that MS is significantly associated with $\mathrm{EO}$ in males but not in females, and NAFLD correlates positively with EO in females but not in males when considering NAFLD and MS simultaneously. Based on the findings from the association between NAFLD severity and EO severity, male participants with MS still exhibited a higher risk for the grade of EO, although different severity of NAFLD was not. In females, mild NAFLD was related to the grade of EO, but MS, moderate and severe NAFLD were not. The discrepancy for the effect of different NAFLD severity on the grade of $\mathrm{EO}$ in females can be explained by the small number of participants with grade $\mathrm{B}-\mathrm{D}$ of $\mathrm{EO}$ or severe NAFLD resulting in an unreliable model for the association between NAFLD severity and EO severity. To the best of our knowledge, this is the first study to reveal significant gender differences in the association of NAFLD and MS with EO.

MS is known to be independently associated with EO via the effects of increased serum cytokines and the volume of visceral fat. ${ }^{6}{ }^{10}$ Elevated serum cytokine levels, such as interleukin 6 (IL-6) and tumour necrosis factor- $\alpha$, can alter neurogenic oesophageal muscle contraction following impaired acid clearance and augmented reflux episodes..$^{31}$ In addition, an increased volume of visceral fat positively correlates with elevated serum cytokine levels ${ }^{6}$ and increased mechanical gastric pressure, causing frequent relaxation of the lower oesophageal sphincter with acid reflux. ${ }^{32}$ NAFLD, known to be associated with obesity and insulin resistance as $\mathrm{MS},{ }^{33}$ was demonstrated to independently correlate with $\mathrm{EO}$ in this study, possibly through the effects of increased serum cytokine levels, visceral fat volume and systemic oxidative stress with a diminished antioxidant capacity. $^{9} 34 \quad 35$ Increased oxidative stress may lead to damage of the adherent mucus layer and oesophageal mucosa,${ }^{36}$ while decreased antioxidant capacity can decrease the ability to prevent oesophageal mucosal damage and accentuate the severity of EO. ${ }^{36}$ 
Several factors could explain why MS is a significant risk factor for EO in males but not in females. First, the patterns of fat distribution differ between males and females. Females more often develop subcutaneous adiposity, whereas males are more prone to visceral fat deposition. ${ }^{37}$ Among participants with MS, males have more visceral fat than subcutaneous fat, while the reverse was observed in females. ${ }^{16}{ }^{17}$ Compared with females, males might suffer more detrimental effects of increased visceral fat volume on EO through elevated serum cytokine levels and mechanically raised gastric pressure. ${ }^{62}$ Moreover, greater visceral adiposity, which is observed more often in males than in females, could result in an increase in serum cytokine secretion to cause EO. ${ }^{38}$ These findings are consistent with a recent study reporting that serum IL-6 and tumour necrosis factor- $\alpha$ levels were higher in males with MS than in females. ${ }^{39}$ Thus, compared with females, males with MS have a higher visceral fat volume and more visceral adiposity, which may result in elevating serum cytokine levels, thereby causing EO. In contrast, the effect of MS on EO was not significant in females with MS, possibly because of a lower visceral fat volume and less visceral adiposity.

The observed gender differences in the relationship between NAFLD and EO may be associated with the effects of oestrogen, which plays a protective role against EO by decreasing serum proinflammatory cytokine levels ${ }^{40}$ and oxidative stress. ${ }^{18}$ One study reported that NAFLD in females correlates with decreased serum oestrogen levels because of impaired mitochondrial and peroxisomal fatty acid $\beta$-oxidation and increased oxidative stress; ${ }^{18}$ hence, the protective effects of oestrogen on EO may be lower in females with NAFLD. Our study also demonstrated that the effect of NAFLD on EO was stronger in premenopausal females than in postmenopausal females. This observation also implied that the serum oestrogen level may play an important role on EO. Furthermore, oestrogen has been associated with less visceral fat volume and smaller visceral adiposity, resulting in lower serum proinflammatory cytokines levels and increased gastric pressure. ${ }^{6} 3238$ This study found no significant increase in the prevalence of EO in females with MS, and this may be because of the effects of oestrogen on body fat distribution and adipocyte size.

This study has several limitations. First, previous studies demonstrated an association between $H$. pylori infection negativity and $\mathrm{EO} ;{ }^{41}$ however, data regarding such an infection were unavailable in this study. Second, data regarding the pattern of fat distribution, serum oestrogen and cytokine levels were also unavailable. Third, since our study participants were restricted to those undergoing self-paid health check-ups, there is a possibility of selection bias. Thus, these results may not apply to the general population. Fourth, the findings might not be generalisable to other ethnic groups because the participants were all Taiwanese. Finally, this study is cross-sectional and thus cannot provide evidence to indicate a causal relationship between NAFLD/MS and EO with respect to gender.

\section{CONCLUSIONS}

Significant gender differences were observed in the effects of NAFLD and MS on EO. The effect of MS on EO severity might be greater than that of NAFLD in males, while the effect of NAFLD on EO severity was greater than that of MS in females. Further studies regarding gender differences in $\mathrm{EO}$ should focus on $\mathrm{EO}$ management and clarifying the relationship between serum oestrogen levels and EO.

\section{Author affiliations}

${ }^{1}$ Department of Family Medicine, E-Da Hospital//-Shou University, Kaohsiung, Taiwan

${ }^{2}$ Department of Family Medicine, National Cheng Kung University Hospital,

Tainan, Taiwan

${ }^{3}$ Department of Family Medicine, College of Medicine, National Cheng Kung University, Tainan, Taiwan

${ }^{4}$ Department of Family Medicine, National Cheng Kung University College of Medicine and Hospital, Dou-Liou Branch, Yunlin, Taiwan

${ }^{5}$ Institute of Gerontology, College of Medicine, National Cheng Kung University, Tainan, Taiwan

Contributors W-CH, J-SW, Y-CY, Z-JS, F-HL and C-JC were involved in the conception and design of the study and interpretation of the data. J-SW and C-JC provided administrative, technical, and material support. F-HL provided technical and material support. All authors were involved in the acquisition of data. W-CH, JSW, Y-CY and C-JC undertook the data analysis. Drafting of the manuscript was done by W-CH, J-SW, Y-CY and C-JC.

Funding This study was supported by the Department of Family Medicine, National Cheng-Kung University Hospital, Taiwan (grant number NCKUHFM-101-003) and E-Da hospital.

Competing interests None declared.

Patient consent Obtained.

Ethics approval The Institutional Review Board of the National Cheng Kung University Hospital (approval number: A-ER-101-317).

Provenance and peer review Not commissioned; externally peer reviewed.

Data sharing statement No additional data are available.

Open Access This is an Open Access article distributed in accordance with the Creative Commons Attribution Non Commercial (CC BY-NC 4.0) license, which permits others to distribute, remix, adapt, build upon this work noncommercially, and license their derivative works on different terms, provided the original work is properly cited and the use is non-commercial. See: http:// creativecommons.org/licenses/by-nc/4.0/

\section{REFERENCES}

1. Goh KL. Gastroesophageal reflux disease in Asia: a historical perspective and present challenges. J Gastroenterol Hepatol 2011;26(Suppl 1):2-10.

2. Ronkainen J, Talley NJ, Storskrubb T, et al. Erosive esophagitis is a risk factor for Barrett's esophagus: a community-based endoscopic follow-up study. Am J Gastroenterol 2011;106:1946-52.

3. Parasa S, Sharma P. Complications of gastro-oesophageal reflux disease. Best Pract Res Clin Gastroenterol 2013;27:433-42.

4. Gunji T, Sato H, lijima K, et al. Risk factors for erosive esophagitis: a cross-sectional study of a large number of Japanese males. J Gastroenterol 2011;46:448-55.

5. Ou JL, Tu CC, Hsu PI, et al. Prevalence and risk factors of erosive esophagitis in Taiwan. J Chin Med Assoc 2012;75:60-4.

6. Chung SJ, Kim D, Park MJ, et al. Metabolic syndrome and viscera obesity as risk factors for reflux oesophagitis: a cross-sectional 
case-control study of 7078 Koreans undergoing health check-ups. Gut 2008:57:1360-5.

7. Park $\mathrm{CH}, \mathrm{Kim} \mathrm{KO}$, Baek IH, et al. Differences in the risk factors of reflux esophagitis according to age in Korea. Dis Esophagus 2014;27:116-21.

8. Wu P, Ma L, Dai GX, et al. The association of metabolic syndrome with reflux esophagitis: a case-control study. Neurogastroenterol Motil 2011;23:989-94.

9. Jang $\mathrm{S}$, Lee $\mathrm{CH}$, Choi KM, et al. Correlation of fatty liver and abdominal fat distribution using a simple fat computed tomography protocol. World J Gastroenterol 2011;17:3335-41.

10. Park JH, Park DI, Kim HJ, et al. Metabolic syndrome is associated with erosive esophagitis. World J Gastroenterol 2008;14:5442-7.

11. Völzke H. Multicausality in fatty liver disease: is there a rationale to distinguish between alcoholic and non-alcoholic origin? World J Gastroenterol 2012;18:3492-501.

12. Altiparmak E, Koklu S, Yalinkilic M, et al. Viral and host causes of fatty liver in chronic hepatitis B. World J Gastroenterol 2005;11: 3056-9.

13. Nascimento AC, Maia DR, Neto SM, et al. Nonalcoholic fatty liver disease in chronic hepatitis $\mathrm{B}$ and $\mathrm{C}$ patients from Western Amazon. Int $J$ Hepatol 2012;2012:695950.

14. Hsu CS, Wang CC, Wang PC, et al. Increased incidence of gastroesophageal reflux disease in patients with chronic hepatitis $B$ virus infection. Hepatol Int 2010;4:585-93.

15. Neuschwander-Tetri BA, Caldwell SH. Nonalcoholic steatohepatitis: summary of an AASLD Single Topic Conference. Hepatology 2003;37:1202-19.

16. Sogabe $\mathrm{M}$, Okahisa $\mathrm{T}$, Kimura $\mathrm{Y}$, et al. Visceral fat predominance is associated with erosive esophagitis in Japanese men with metabolic syndrome. Eur J Gastroenterol Hepatol 2012;24: $910-16$

17. Sogabe M, Okahisa T, Tsujigami K, et al. Visceral fat predominance is associated with non-alcoholic fatty liver disease in Japanese women with metabolic syndrome. Hepatol Res 2014;44:515-22.

18. Gutierrez-Grobe $\mathrm{Y}$, Ponciano-Rodriguez $\mathrm{G}$, Ramos $\mathrm{MH}$, et al. Prevalence of non alcoholic fatty liver disease in premenopausal, postmenopausal and polycystic ovary syndrome women. The role of estrogens. Ann Hepatol 2010;9:402-9.

19. Hung WC, Wu JS, Yang YC, et al. Nonalcoholic fatty liver disease vs. obesity on the risk of erosive oesophagitis. Eur J Clin Invest 2014:44:1143-9.

20. Husten CG. How should we define light or intermittent smoking? Nicotine Tob Res 2009;11:111-21.

21. WHO. Report of a WHO Consultation on Obesity: Prevention and Managing, the Global Epidemic. Geneva: World Health Organization, 1999.

22. Pan WH, Lee MS, Chuang SY, et al. Obesity pandemic, correlated factors and guidelines to define, screen and manage obesity in Taiwan. Obes Rev 2008:9(Suppl 1):22-31.

23. WHO. International Association for the Study of Obesity, International Obesity Task Force: the Asia-Pacific Perspective: redefining obesity and its treatment. Sydney: Health Communications, 2000

24. Lenfant $\mathrm{C}$, Chobanian $\mathrm{AV}$, Jones DW, et al., Joint National Committee on the Prevention, Detection, Evaluation, and Treatment of High Blood Pressure. Seventh report of the Joint National Committee on the Prevention, Detection, Evaluation, and Treatment of High Blood Pressure (JNC 7): resetting the hypertension sails. Hypertension 2003:41:1178-9.

25. Levey AS, Bosch JP, Lewis JB, et al. A more accurate method to estimate glomerular filtration rate from serum creatinine: a new prediction equation. Modification of Diet in Renal Disease Study Group. Ann Intern Med 1999;130:461-70.

26. American Diabetes Association. Diagnosis and classification of diabetes mellitus. Diabetes Care 2011;34(Suppl 1):S62-9.

27. Grundy SM, Cleeman JI, Daniels SR, et al. Diagnosis and management of the metabolic syndrome: an American Heart Association/National Heart, Lung, and Blood Institute Scientific Statement. Circulation 2005;112:2735-52.

28. Saverymuttu SH, Joseph AE, Maxwell JD. Ultrasound scanning in the detection of hepatic fibrosis and steatosis. BMJ 1986;292: 13-15.

29. Armstrong D, Bennett JR, Blum AL, et al. The endoscopic assessment of esophagitis: a progress report on observer agreement. Gastroenterology 1996;111:85-92.

30. Johnson DA, Younes Z, Hogan WJ. Endoscopic assessment of hiatal hernia repair. Gastrointest Endosc 2000;52:650-9.

31. Rieder F, Biancani $\mathrm{P}$, Harnett $\mathrm{K}$, et al. Inflammatory mediators in gastroesophageal reflux disease: impact on esophageal motility, fibrosis, and carcinogenesis. Am J Physiol Gastrointest Liver Physiol 2010;298:G571-81.

32. Wu JC, Mui LM, Cheung CM, et al. Obesity is associated with increased transient lower esophageal sphincter relaxation. Gastroenterology 2007;132:883-9.

33. McCullough AJ. Pathophysiology of nonalcoholic steatohepatitis. $J$ Clin Gastroenterol 2006;40(Suppl 1):S17-29.

34. Wieckowska A, Papouchado BG, Li Z, et al. Increased hepatic and circulating interleukin-6 levels in human nonalcoholic steatohepatitis Am J Gastroenterol 2008;103:1372-9.

35. Kumar R, Prakash S, Chhabra S, et al. Association of pro-inflammatory cytokines, adipokines \& oxidative stress with insulin resistance \& non-alcoholic fatty liver disease. Indian J Med Res 2012;136:229-36.

36. Oh TY, Lee JS, Ahn BO, et al. Oxidative stress is more important than acid in the pathogenesis of reflux oesophagitis in rats. Gut 2001;49:364-71.

37. Williams CM. Lipid metabolism in women. Proc Nutr Soc 2004;63:153-60.

38. Pradhan AD. Sex differences in the metabolic syndrome: implications for cardiovascular health in women. Clin Chem 2014;60:44-52.

39. Bălăşoiu M, Bălăşoiu AT, Stepan AE, et al. Proatherogenic adipocytokines levels in metabolic syndrome. Rom J Morphol Embryol 2014;55:29-33.

40. Tani A, Yasui T, Matsui S, et al. Circulating levels of monocyte chemoattractant protein-1 and interleukin-7 in women who have undergone bilateral salpingo-oophorectomy. J Inflamm Res 2013;7:1-7.

41. Koike T, Ohara S, Sekine $H$, et al. Helicobacter pylori infection prevents erosive reflux oesophagitis by decreasing gastric acid secretion. Gut 2001:49:330-4. 